\title{
Variation on a Zernike wavefront sensor theme: Optimal use of photons
}

\author{
V. Chambouleyron ${ }^{1,2}$, O. Fauvarque ${ }^{3}$, J-F. Sauvage ${ }^{2,1}$, K. Dohlen $^{1}$, N. Levraud ${ }^{2,1}$, A. Vigan ${ }^{1}$, M. N'Diaye ${ }^{4}$, \\ B. Neichel ${ }^{1}$, and T. Fusco ${ }^{2,1}$ \\ 1 Aix Marseille Univ, CNRS, CNES, LAM, Marseille, France \\ e-mail: vincent. chambouleyron@lam. fr \\ 2 DOTA, ONERA, Université Paris Saclay, 91123 Palaiseau, France \\ 3 IFREMER, Laboratoire Detection, Capteurs et Mesures (LDCM), Centre Bretagne, ZI de la Pointe du Diable, CS 10070, \\ 29280 Plouzane, France \\ ${ }^{4}$ Université Côte d'Azur, Observatoire de la Côte d'Azur, CNRS, Laboratoire Lagrange, France
}

Received 24 March 2021 / Accepted 17 May 2021

\begin{abstract}
Aims. The Zernike wavefront sensor (ZWFS) is a concept belonging to the wide class of Fourier-filtering wavefront sensors (FFWFSs). The ZWFS is known for its extremely high sensitivity and low dynamic range, which makes it a unique sensor for second stage adaptive optics systems or quasi-static aberration calibration sensors. This sensor is composed of a focal plane mask made of a phase shifting dot that is fully described by two parameters: its diameter and depth. We aim to improve the performance of this sensor by changing the diameter of its phase shifting dot.

Methods. We begin with a general theoretical framework, providing an analytical description of the FFWFS properties. We then predict the expected ZWFS sensitivity for different configurations of dot diameters and depths. The analytical predictions are then validated with end-to-end simulations. From this, we propose a variation of the classical ZWFS shape that exhibits extremely appealing properties.

Results. We show that the ZWFS sensitivity can be optimized by modifying the dot diameter and it can even reach the optimal theoretical limit, though with the trade-off of low spatial frequency sensitivity. As an example, we show that a ZWFS with a $2 \lambda / D$ dot diameter (where $\lambda$ is the sensing wavelength and $D$ the telescope diameter), hereafter called a Z2WFS, exhibits a sensitivity twice higher than the classical 1.06 $\lambda / D$ ZWFS for all the phase spatial components except for tip-tilt modes. Furthermore, this gain in sensitivity does not impact the dynamic range of the sensor, and the Z2WFS exhibits a similar dynamical range as the classical $1.06 \lambda / D$ ZWFS. This study opens the path to the conception of a diameter-optimized ZWFS.
\end{abstract}

Key words. instrumentation: adaptive optics - telescopes

\section{Introduction}

The role of a wavefront sensor (WFS) is to encode the phase information at the entrance of an optical system into intensities on a detector. For ground-based astronomy, WFSs are mostly used for active or adaptive optics (AO) in conjunction with a wave-front control strategy in order to compensate for optical aberrations induced by the atmosphere or the telescope itself. In the context of astronomy, one of the main drivers for a WFS design is its sensitivity, or in other words, its ability to provide an accurate measurement in the presence of noise. Sensitivity is therefore a useful metric for assessing WFS performance in terms of photon noise, which is related to key quantities in the AO field: loop speed and sky-coverage. Existing WFSs can be separated into two main categories, usually defined as focal plane WFSs, for which the measurements are done in a focal plane (such as the Shack-Hartmann WFS), and pupil plane WFSs, for which the measurements are done in a pupil plane. Among this latter category, the Fourier filtering WFS (FFWFS) represents a wide class of sensors of particular interest thanks to their superior sensitivity. From a general point of view, an FFWFS consists of a phase mask located in an intermediate focal plane that performs an optical Fourier filtering. As such, the Zernike phase mask (Zernike \& Stratton 1934; Bloemhof \& Wallace 2003; Dohlen et al. 2006; Wallace et al. 2011) forms an FFWFS, hereafter a Zernike WFS (ZWFS). In this case, the filtering element is a phase shifting dot that is, for a given substrate, fully described by two parameters: its diameter and its depth (or phase shift). In a classical implementation, the ZWFS phase dot has a diameter of $1.06 \lambda / D$ (where $\lambda$ is the sensing wavelength and $D$ the telescope diameter) and a phase shift of $\pi / 2$. This ZWFS is known to be one of the most sensitive WFSs (Guyon 2005). Its drawback being its limited dynamic range, it has therefore been mostly implemented as a second-stage WFS or as a quasi-static aberration calibration sensor, such as on VLT/SPHERE (N'Diaye et al. 2016; Vigan et al. 2019). In this paper we show that the classical implementation of the ZWFS with a phase dot diameter of $1.06 \lambda / D$ is actually not optimal: by using a larger dot diameter, the sensitivity of the ZWFS can be significantly improved, at the expense of lower spatial frequency sensitivity, and can even reach a performance close to the theoretical limit. For this reason, Sect. 2 starts 
from a theoretical study of the ZWFS based on a general convolutional formalism for FFWFSs (Fauvarque et al. 2016). This analytical work shows that the sensitivity of the ZWFS can be improved by increasing its dot diameter. We then confirm the theoretical results with end-to-end simulations in Sect. 3, and we show that a gain in sensitivity by a factor of two can be reached without impacting the dynamic range of the sensor. Conclusions are given in Sect. 4.

\section{Theoretical analysis of the ZWFS sensitivity with a convolutional approach}

\subsection{Definition of FFWFS sensitivity}

Following the formalism introduced by Fauvarque et al. (2016), the raw intensities recorded by an FFWFS are processed with a return-to-reference operation. This simply consists in removing from the FFWFS recorded intensities map $I(\phi)$ the one corresponding to the reference phase $I_{0}$ (usually a flat wavefront). We also posit that all the intensities are normalized by the number of photons. The resulting quantity is called the tared intensities:

$$
\Delta I(\phi)=I(\phi)-I_{0}
$$

One of the most important performance criteria for a sensor is its behavior in terms of noise propagation. This criteria is encoded in a quantity called sensitivity, which depends on the energy in the columns of the interaction matrix (IM; Rigaut \& Gendron 1992). For a given WFS, each column of the IM is built as the linear response of the sensor to a given mode $\phi_{i}$, which is usually obtained experimentally through a "push-pull" method:

$\delta I\left(\phi_{i}\right)=\frac{I\left(\epsilon \phi_{i}\right)-I\left(-\epsilon \phi_{i}\right)}{2 \epsilon}$,

where $\epsilon$ is the amplitude of the mode. The sensitivity $s$ for a given mode $\phi_{i}$ is then defined through the Euclidean norm:

$s\left(\phi_{i}\right)=\frac{\left\|\delta I\left(\phi_{i}\right)\right\|_{2}}{\left\|\phi_{i}\right\|_{2}}$.

For a given uniform noise distribution $\sigma_{\mathrm{n}}$, the noise propagation coefficient $\sigma_{\text {WFS }}$ for a mode $\phi_{i}$ is then related to the sensitivity by the following relationship:

$\sigma_{\mathrm{WFS}}^{2}=\sigma_{\mathrm{n}}^{2} s\left(\phi_{i}\right)^{-2}$

For ground-based astronomy, where WFSs are usually implemented within an AO loop, the sensitivity is a critical metric as it describes how the system performs in the presence of noise. Optimizing the WFS sensitivity has always been one of the main motivations in the conception of new WFSs.

Finally, it should be noticed that we chose to visualize the FFWFS sensitivity as a two-dimensional map along the spatial frequencies of the wavefront. This consisted in calculating sensitivity with respect to the Fourier modes $\phi_{i}$ (close to what was done in Jensen-Clem et al. 2012), which are simply defined by the sum of a cosine and a sine carrying a given spatial frequency $f$. The following quantity then encodes the sensitivity:

$s_{f}=\sqrt{s\left(\cos _{f}\right)^{2}+s\left(\sin _{f}\right)^{2}}$.

\subsection{A convolutional approach to computing FFWFS sensitivity}

The FFWFS sensitivity can be computed based on a convolutional model, as described in Fauvarque et al. (2019). This model assumes that the sensor can be fully characterized by an impulse response IR that links the entrance phase to the measured tared intensities:

$\Delta I(\phi) \approx \mathrm{IR} \star \phi$,

where $\star$ stands for the classical convolutional product. A convenient aspect of the convolutional approach is the fact that one can compute the transfer function TF of an FFWFS. FFWFSs can be described by two parameters: their phase masks $m$ and their weighting functions $\omega$, which describe the energy distribution in the focal plane during one acquisition time of the sensor. We note that this function is normalized to 1 in order to ensure energy conservation. Assuming that $\omega$ is a real function and that $\omega$ and $m$ are both centro-symmetric, which is generally the case for most of the known FFWFSs, TF is expressed through the following simple formula:

$\mathrm{TF}=2 \operatorname{Im}[m \star \overline{m \omega}]$,

where $\mathrm{Im}$ is the imaginary part and the bar is the complex conjugate operator. From the knowledge of an FFWFS transfer function, it is then possible to compute the sensitivity with respect to spatial frequencies thanks to the formula given in Fauvarque et al. (2019):

$\left.s_{f} \approx \sqrt{|\mathrm{TF}|^{2} \star \mathrm{PSF}}\right|_{f}$,

where the quantity PSF is the point spread function of the system. Its energy corresponds to the incoming flux and is normalized to 1 .

At this point, it is important to note that the sensitivity is bounded. Since the mask transmission, $|m|$, cannot be greater than $1(|m| \leq 1)$, Eq. (7) implies that $\left.\mathrm{TF}\right|_{f} \leq 2$. Hence, given Eq. (8), we conclude that, in the frame of our normalizations, the sensitivity cannot be greater than 2 :

$\forall f, s_{f} \leq 2$.

This is an important result as it defines the theoretical limit for the sensitivity of an FFWFS.

\subsection{Application to the ZWFS}

The convolutional formalism introduced in the previous section was then applied to the ZWFS in order to find a simple formula of its sensitivity according to the mask parameters. As previously mentioned, the ZWFS mask is defined by two free parameters. The first is the depth (phase shift) of the dot $\delta$; for the classical ZWFS, $\delta=\pi / 2$. The second is its diameter $p$; for the classical ZWFS, $p=1.06 \lambda / D$. As described in N'Diaye et al. (2013), the dot diameter value was chosen in order to get an equivalent flux inside and outside the focal plane dot. This configuration with $p=1.06 \lambda / D$ also allows a uniform reference intensity distribution to be obtained. The purpose of this section is to demonstrate that this choice of $p=1.06 \lambda / D$ is actually not optimal, and that the sensitivity can be improved with a larger dot.

For the sake of clarity, we carried out this study in one dimension. As such, the spatial frequency vector $f$ becomes the scalar frequency $f$. We further assumed that the weighting function $\omega$, 

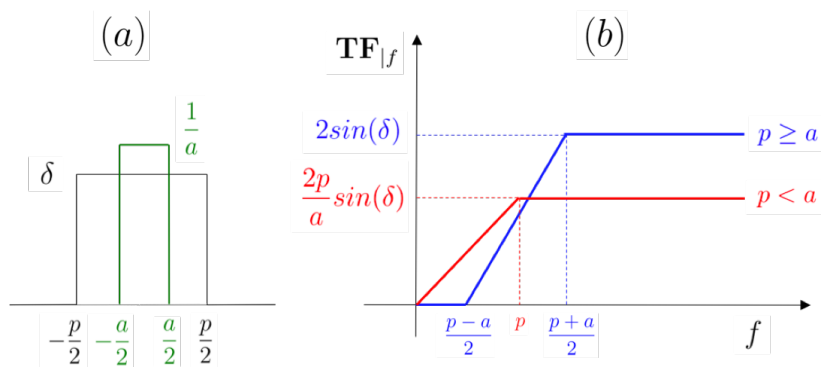

Fig. 1. Simplified description of the ZWFS. (a): simplified onedimensional framework for convolutional derivations; in black, the dot diameter is equal to $p$ and the phase shift is equal to $\delta$. In green, the PSF is approximated by a normalized top-hat function with a diameter $a$. (b): transfer function of the ZWFS for two cases; in red the dot size is smaller than the PSF, and in blue the dot is larger than the PSF. The optimal case appears for $p=a$ and not $p=a / 2$, as done for the classical ZWFS.

which corresponds to the PSF, can be described as a top-hat function of diameter radius $a$. This simplified geometry is summarized by Fig. 1a. From this simplified geometry, we then computed $\left.\mathrm{TF}\right|_{f}$ and plot this quantity in Fig. 1b. The full derivation can be found in Appendix B.

From Fig. 1b one can distinguish two cases. The first is $p \geq a$ (i.e., the dot diameter is larger than the PSF characteristic size). Frequencies above $(p+a) / 2$ reach the sensitivity $2 \sin (\delta)$. Frequencies below $(p-a) / 2$ are at 0 . The second is $p<a$ : the dot diameter is smaller than the PSF size. Frequencies over $p$ have a value of $2 p / a \times \sin (\delta)$, which is smaller than the theoretical limit of 2.

From this simplified model one can first conclude that a phase shift of $\delta=\pi / 2$ will maximize the sensitivity, as expected. But surprisingly, it shows that for this phase shift a dot radius of $p=a$ offers a sensitivity of the optimal value for almost all the modes. At this point it is important to remember that the value of $\mathrm{TF}_{f}$ directly sets the sensor sensitivity through Eq. (8). It is therefore possible to design a ZWFS that reaches the theoretical sensitivity value. As a comparison, the classical ZWFS configuration (N'Diaye et al. 2013) uses $p=a / 2$, which leads to a suboptimal TF value of 1 for frequencies above $p$.

Although this simplified study uses strong assumptions, it shows that the ZWFS can be further optimized compared to its classical form. In the next section we demonstrate that these simplified results are accurate and will enable us to build the most sensitive sensor ever proposed.

\section{Toward an optimal ZWFS}

Following the results from the convolutional approach, the goal of this section is to make use of numerical simulations to confirm the sensitivity of the ZWFS with respect to its dot diameter, and eventually to propose an optimal configuration. For that, we consider different configurations for a dot diameter ranging from 0 to $5 \lambda / D$. The phase shift is set to $\delta=\pi / 2$ for the rest of this Letter.

\subsection{Impact of the dot diameter on the ZWFS sensitivity}

As a first step, we want to illustrate the impact of the dot diameter on sensitivity for a spatial frequency located outside of the dot (horizontal part of the curves in Fig. 1b). For that purpose, we arbitrary chose a spatial frequency with six cycles over the pupil (left insert of Fig. 2), which is far enough from the
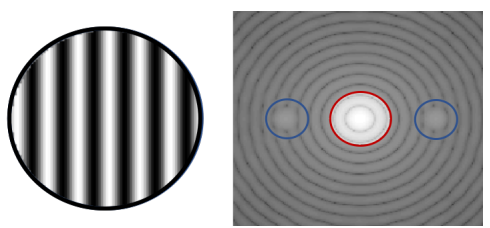

Fig. 2. Cosine phase (left insert, linear scale) corresponding to a spatial frequency of six cycles in D and its corresponding PSF speckles (right insert, logarithmic scale, in blue). This frequency lies outside of the dot footprint, here $5 \lambda / D$ (in red).

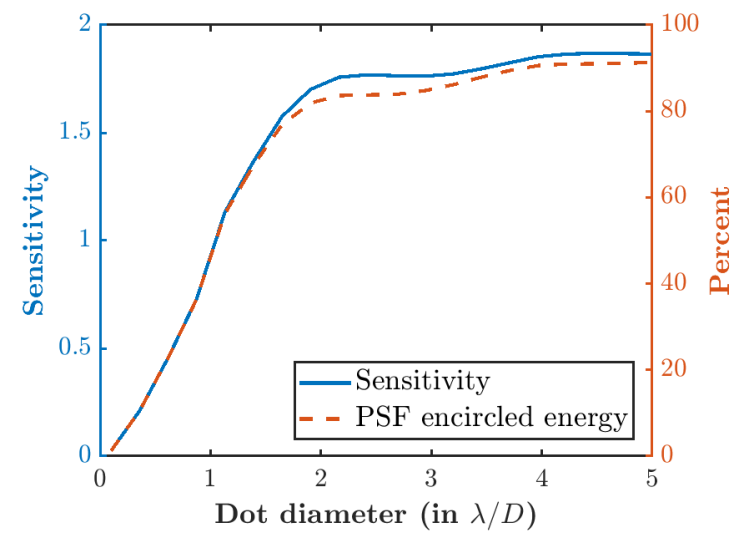

Fig. 3. Sensitivity evolution for a frequency outside of the dot with increasing dot diameter. The sensitivity is in strong accordance with the proportion of the PSF energy located inside the dot, as predicted by the convolutional approach.

maximum diameter dot value $(5 \lambda / D$, i.e., a radius of $2.5 \lambda / D)$. This configuration is illustrated in the right insert of Fig. 2. The sensitivity results for this spatial frequency are shown in Fig. 3.

As predicted by the convolutional approach, Fig. 3 shows that the sensitivity for a high spatial frequency increases with the dot diameter. This behavior has been discussed briefly in previous literature (Ruane et al. 2020) without further analysis. It is explained here thanks to the convolutional model. It is also interesting to notice that the sensitivity growth closely follows the PSF encircled energy in the dot diameter, confirming the analytical results presented in Fig. 1. For a classical ZWFS, with a dot diameter of $1.06 \lambda / D$, the sensitivity is actually far from optimal.

However, one cannot just increase the dot diameter inconsiderately because the sensitivity to frequencies lying inside the dot would drop to 0 (Fig. 1). We illustrate this effect in Fig. 4, where we plot the sensitivity curves with respect to a wide range of spatial frequencies for the three dot diameter configurations $p=1.06,2$, and $5 \lambda / D$. For $p=5 \lambda / D$, the sensitivity to high-spatial frequencies (those larger than five cycles per pupil) almost reaches the theoretical limit of 2; however, the sensitivity becomes close to 0 for low-spatial frequencies (those smaller than three cycles per pupil). There is therefore a trade-off between enhanced sensitivity and unseen modes. In the following, we propose the configuration with $p=2 \lambda / D$ and call this specific configuration the Z2WFS.

As a remark, we also emphasize that the reference intensities (i.e., the intensity distribution for a flat wavefront) change with the value of $p$. This is illustrated in Fig. 5 for the previous three different values of $p$. The classical ZWFS shows a flat reference illumination, while the Z2WFS appears to be less uniform. This spatial distribution could involve practical issues in terms of detector dynamics or for complex pupil shapes, such as 


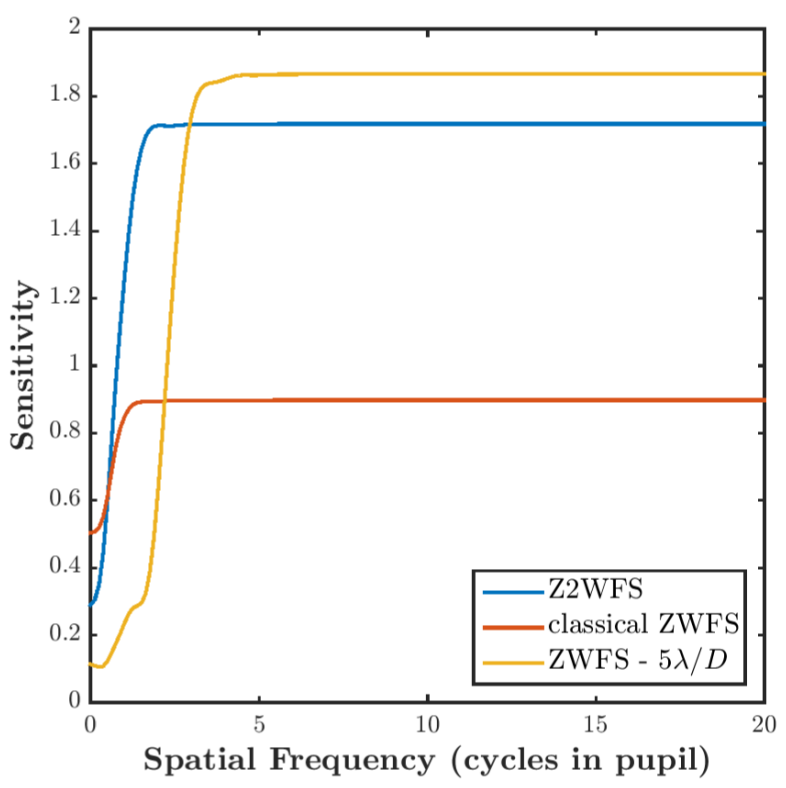

Fig. 4. Sensitivity curves for different dot diameters. This figure has to be compared with the convolutional approach in Fig. 1.
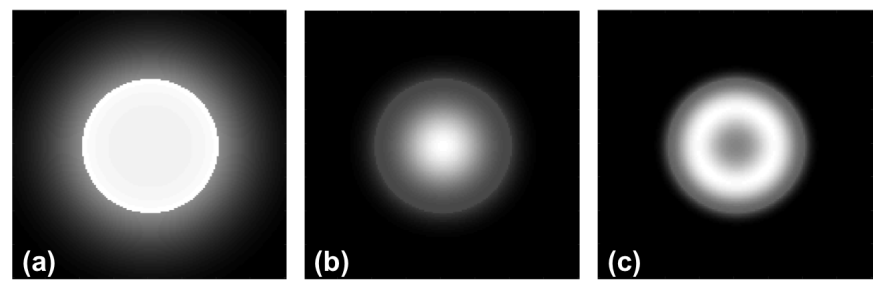

Fig. 5. Reference intensities of the ZWFS for different dot diameters. (a) $p=1.06 \lambda / D$. (b) $p=2 \lambda / D$. (c) $p=5 \lambda / D$.

central obscuration or spiders. These potential practical implementation issues are beyond the scope of this paper. In this Letter we only assume a full aperture pupil with monochromatic light for the sake of clarity. It is to be noted that there is no sticking point here; the formalism and results developed here are maintained with a central obscuration in the pupil.

\subsection{Comparison with other FFWFSs}

In this section we compare the Z2WFS with other well-know FFWFSs: the classical ZWFS with $p=1.06 \lambda / D$, the nonmodulated pyramid WFS (PyWFS) (Ragazzoni 1996), the modulated PyWFS (here with a modulation radius of $3 \lambda / D$ ), and a flattened pyramid WFS (FPyWFS) proposed by Fauvarque et al. (2015) with a pupil overlapping rate of $75 \%$. Spatial frequencies basis (i.e., Fourier basis) was chosen for this comparison. Results are given in Fig. 6. First, we retrieved well-know results, such as the gain of around a factor of two in sensitivity between the classical ZWFS and the PyWFS. We can also highlight the behavior of the FPyWFS, which shows oscillating sensitivity and peaks for some specific frequencies, as described in Fauvarque et al. (2015). (The explanation of the PyWFS class behavior through to the convolutional approach is also given in Appendix C.) The Z2WFS is clearly the most sensitive sensor, except for extremely low frequencies. As expected from Fig. 3, it has a sensitivity twice better than the classical ZWFS for almost all modes and is four times more sensitive than the non-modulated PyWFS.

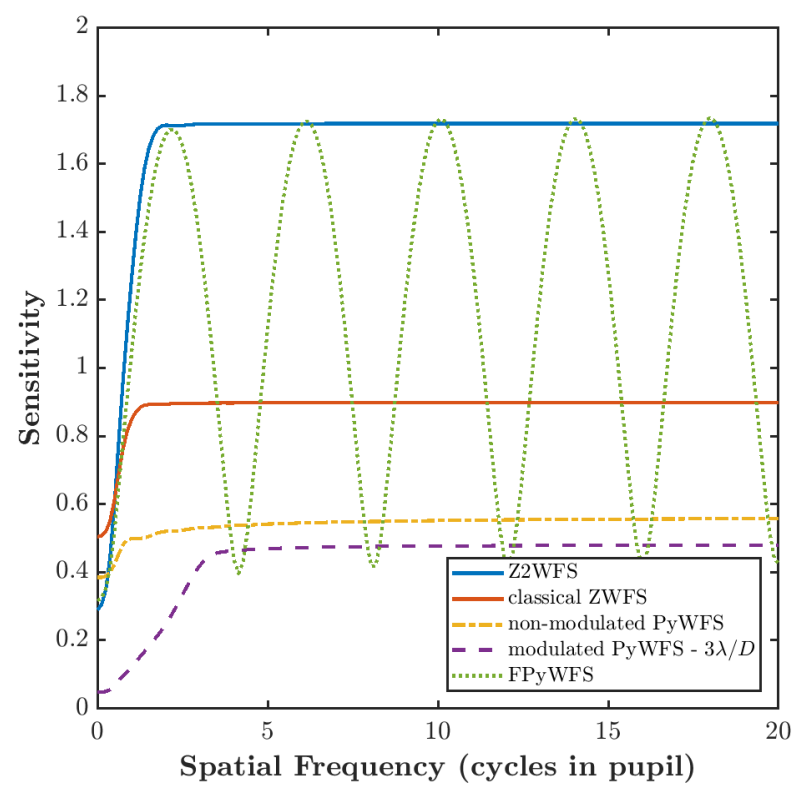

Fig. 6. Sensitivity curves for different FFWFSs. We can distinguish the ZWFS class and the PyWFS class. The Z2WFS overtakes all the other sensors.

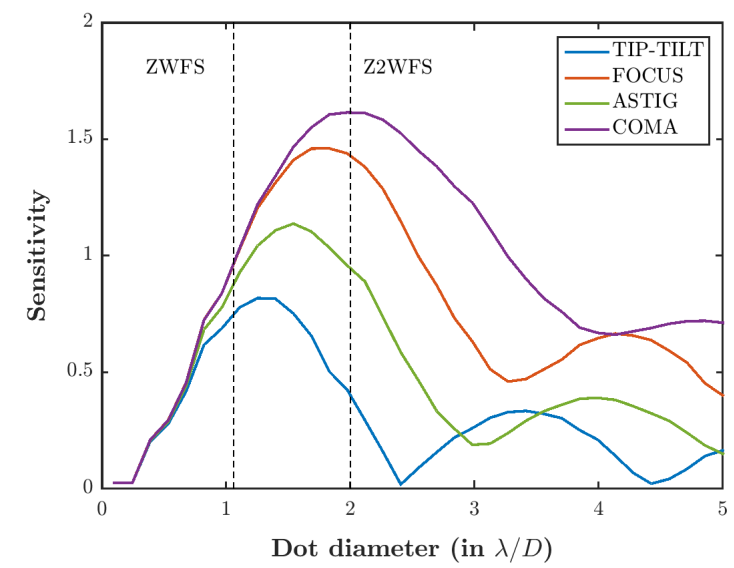

Fig. 7. Evolution of the low order Zernike mode sensitivities with respect to the dot diameter. We can see that the Z2WFS sensitivity is lower for the tip-tilt modes but higher for all the others. The classical ZWFS is not even optimized for the tip-tilt modes.

The behavior at low spatial frequencies deserves some further analysis: we plot in Fig. 7 the sensitivity with respect to the tip-tilt and focus modes (which are the lowest frequency Zernike modes) for the ZWFS class with a dot diameter ranging from 0 to $5 \lambda / D$. For the tip-tilt modes, the Z2WFS is half as sensitive as the classical ZWFS, but the Z2WFS provides better results for the focus. Even if the Z2WFS is less sensitive for tip-tilt than the classical ZWFS, it is important to note that it remains as sensitive as the non-modulated PyWFS, which is around 0.4. As a remark, it is interesting to see that the sensitivity curve for the tip-tilt follows the PSF shape: for the edge of the dot lying on a dark area of the PSF, the sensitivity drops to 0 . By taking a Z1.5WFS ( $p=1.5 \lambda / D)$, one could have a better sensitivity for all the frequencies compared to the classical ZWFS, but a lower gain overall compared to a Z2WFS.

We have demonstrated that a Z2WFS has significantly improved sensitivity and approaches the ideal FFWFS behavior. When compared to the classical ZWFS, the gain in sensitivity 


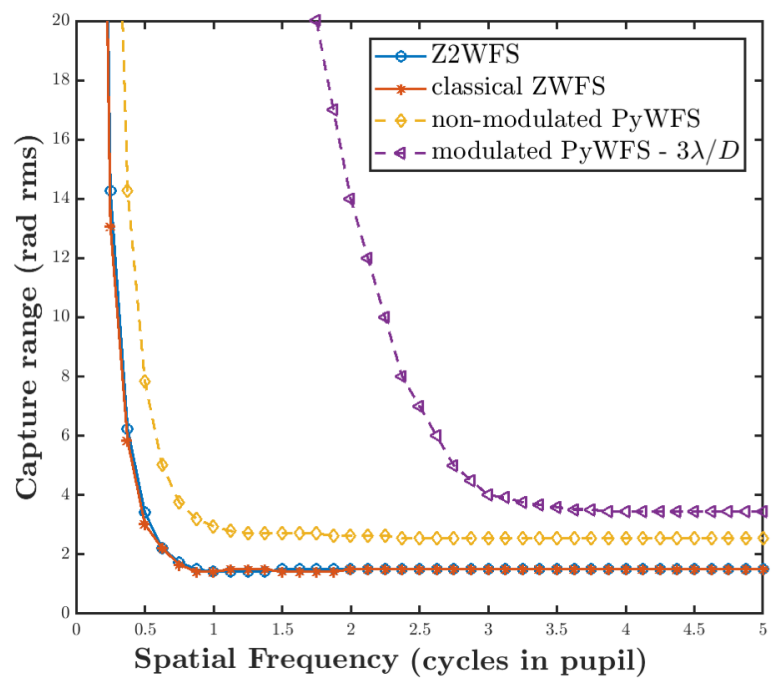

Fig. 8. Capture range of different FFWFSs. The Z2WFS has the same capture range as the classical ZWFS.

for all modes, except the tip-tilt, is a factor of two. In the next section we investigate if this gain in sensitivity comes at the cost of dynamic range.

\subsection{Dynamic range}

To complete our study we now compare the dynamic range of the Z2WFS with the classical ZWFS. A drastic loss in dynamic range with increasing dot diameter could indeed prevent a practical utilization of the Z2WFS. To calculate this quantity with respect to a given mode $\phi_{i}$, we evaluated its capture range $C_{\phi_{i}}$. To calculate it, we looked at the lowest amplitude value (in absolute value) such that:

$$
\left.\frac{\mathrm{d} \Delta I\left(a \phi_{i}\right)}{\mathrm{d} a}\right|_{a_{0}}=0 .
$$

We then defined the capture range as $C_{\phi_{i}}=2 a_{0}$, where the factor 2 allows one to take negative and positive amplitudes into account in the capture range calculation.

The capture range can be larger than the pure linearity regime. However, we decided to use this definition for two reasons: first because it defines the amplitude below which we are ensured that a closed loop system will eventually converge. Indeed, even if the measurement is no longer linear, there is still a one-to-one correspondence with the input signal. Secondly, the ZWFS measurements are often processed through nonlinear reconstructors (N'Diaye et al. 2013; Steeves et al. 2020) that can be perfectly applied to a Z2WFS or other variations of the ZWFS.

Capture range values for cosine phase modes at frequencies ranging from zero to five cycles in diameter are given in Fig. 8 for Zernike and python WFSs. The PyWFS class has a better capture range over all spatial frequencies, matching the fact that sensitivity and dynamic range are competing properties. This graph also confirms the great benefit in terms of dynamic range provided by the modulation of the PyWFS. More importantly, we see that the Z2WFS exhibits the same capture range as the classical ZWFS for high frequencies and is even higher for the lowest frequencies where the Z2WFS sensitivity falls below that of the classical ZWFS. The Z2WFS is therefore more sensitive than the classical ZWFS while exhibiting the same capture range.

\section{Conclusion}

In this paper we have provided a physical description of the sensitivity behavior for the ZWFS class, and in particular we have studied the sensitivity evolution for different dot diameters. We have shown that it is possible to significantly improve the current sensitivity of the ZWFS, at the expense of the lower spatial frequencies, simply by increasing the dot diameter. The resulting sensitivity can even almost reach the fundamental limit of FFWFSs. We further studied the specific case of a dot diameter of $2 \lambda / D$, called a Z2WFS, which exhibits an average factor of two gain in sensitivity (with a loss in sensitivity compared to the classical ZWFS only for the tip-tilt modes), without any loss in the dynamic range with respect to the classical ZWFS. This new sensor thus becomes the most sensitive WFS available for ground-based astronomy. It still exhibits the low dynamic range of the ZWFS, but, as for the PyWFS, modulation schemes can be imagined. For instance, one way to increase its linearity range is to dynamically change the dot diameter during one integration time of the sensor camera. Future studies will investigate its practical implementation and the impact of chromaticity on wavefront sensing.

Acknowledgements. This work benefited from the support of the WOLF project ANR-18-CE31-0018 of the French National Research Agency (ANR). It has also been prepared as part of the activities of OPTICON H2020 (2017-2020) Work Package 1 (Calibration and test tools for AO assisted E-ELT instruments). OPTICON is supported by the Horizon 2020 Framework Programme of the European Commission's (Grant number 730890). Authors are acknowledging the support by the Action Spécifique Haute Résolution Angulaire (ASHRA) of CNRS/INSU co-funded by CNES. Vincent Chambouleyron $\mathrm{PhD}$ is co-funded by "Région Sud" and ONERA, in collaboration with First Light Imaging. AV acknowledges funding from the European Research Council (ERC) under the European Union's Horizon 2020 research and innovation programme (grant agreement No. 757561). Finally, part of this work is supported by the LabEx FOCUS ANR11-LABX-0013, and received the support of Action Spécifique Haute Résolution Angulaire ASHRA.

\section{References}

Bloemhof, E. E., \& Wallace, J. K. 2003, in Astronomical Adaptive Optics Systems and Applications, eds. R. K. Tyson, \& M. Lloyd-Hart, SPIE Conf. Ser., 5169, 309

Dohlen, K., Langlois, M., \& Lanzoni, P. 2006, in Ground-based and Airborne Telescopes, ed. L. M. Stepp, SPIE, 6267, 1093

Fauvarque, O., Neichel, B., Fusco, T., \& Sauvage, J.-F. 2015, Opt. Lett., 40, 3528 Fauvarque, O., Neichel, B., Fusco, T., Sauvage, J.-F., \& Girault, O. 2016, Optica, 3,1440

Fauvarque, O., Janin-Potiron, P., Correia, C., et al. 2019, J. Opt. Soc. Am. A, 36, 1241

Guyon, O. 2005, ApJ, 629, 592

Jensen-Clem, R., Wallace, J. K., \& Serabyn, E. 2012, in 2012 IEEE Aerospace Conference, 1

N'Diaye, M., Dohlen, K., Fusco, T., \& Paul, B. 2013, A\&A, 555, A94

N'Diaye, M., Vigan, A., Dohlen, K., et al. 2016, SPIE Astron. Telescopes Instr., 9909, 99096S

Ragazzoni, R. 1996, J. Mod. Opt., 43, 289

Rigaut, F., \& Gendron, E. 1992, A\&A, 261, 677

Ruane, G., Wallace, J. K., Steeves, J., et al. 2020, J. Astron. Telescopes Instr. Syst., 6, 045005

Steeves, J., Wallace, J. K., Kettenbeil, C., \& Jewell, J. 2020, Optica, 7, 1267

Vérinaud, C. 2004, Opt. Commun., 233, 27

Vigan, A., N'Diaye, M., Dohlen, K., et al. 2019, A\&A, 629, A11

Wallace, J. K., Rao, S., Jensen-Clem, R. M., \& Serabyn, G. 2011, in Optical Manufacturing and Testing IX, eds. J. H. Burge, O. W. Fähnle, \& R. Williamson, SPIE, 8126, 110

Zernike, F., \& Stratton, F. 1934, MNRAS, 94, 377 


\section{Appendix A: Convolutional approach: General framework}

In the infinite pupil approximation and assuming that the weighting function (energy distribution at the focal plane) $\omega$ is a real centro-symmetric function and that the focal-plane mask function $m$ is also centro-symmetric, the transfer function of an FFWFS may be written as:

$\mathrm{TF}=2 \operatorname{Im}[m \star \overline{m \omega}]$.

We only consider in this formalism a pure phase mask for the Fourier filter function $m$ because the global context is the search for WFS sensitivity; as such, we did not consider amplitudemask, which would result in a waste of photons. Therefore, they are pure phase masks that can be written as $m=e^{i \Delta}$. Moreover, we continued to carry out these mathematical developments in one dimension. The previous Eq. (A.1) becomes:

$\left.\mathrm{TF}\right|_{f}=\left.2 \int_{\mathbb{R}} \mathrm{d} u \omega\right|_{u} \sin \left(\left.\Delta\right|_{u}-\left.\Delta\right|_{f-u}\right)$,

where $u$ is expressed in units of $\lambda / D$. We chose to approximate the weighting function as a rectangular and normalized function with a diameter $a$ :

$\left.\omega\right|_{u}=\left.\frac{1}{a} \mathbb{I}_{[-a / 2 ; a / 2]}\right|_{u}$.

The normalization allows one to respect the energy conservation. Furthermore, $a$ may be seen as the characteristic size of the energy distribution at the focal plane. Here, $a$ is therefore the typical size of the modulation of the PyWFS class and corresponds to the PSF characteristic length when modulation is inactive. In other words, the weighting function allows us to take the finite size of the pupil into account despite the "infinite pupil approximation" needed in the convolutional approach.

\section{Appendix B: Zernike WFS class}

For the ZWFS, we consider the following mask: a centered dot with a diameter of $p$ and a depth $\delta$. Thus, the phase of the filtering mask equals:

$\left.\Delta\right|_{u}=\left.\delta \mathbb{I}_{[-p / 2 ; p / 2]}\right|_{u}$.

The problem being symmetric, the derivation of Eq. (A.2) is only done for positive frequencies, $f \geq 0$. We can distinguish two cases: when the size of the dot is bigger than the PSF (i.e., $p \geq a$ ) or not (i.e., $p<a$ ).

Case 1. The dot is larger than the PSF: $p \geq a$.

Case $1.1 f \geq \frac{p+a}{2}$.

We have $\left.\Delta\right|_{u}=\delta$ and $\left.\Delta\right|_{f-u}=0$.

$\left.\mathrm{TF}\right|_{f}=\frac{2}{a} \int_{-a / 2}^{a / 2} \mathrm{~d} u \sin \left(\left.\Delta\right|_{u}-\left.\Delta\right|_{f-u}\right)=\frac{2}{a} \int_{-a / 2}^{a / 2} \mathrm{~d} u=2 \sin (\delta)$.

Case $1.2 f<\frac{p+a}{2}$.

In this case, $\left.\Delta\right|_{u}{ }^{2}-\left.\Delta\right|_{f-u}$ depends on $f$. We get:

$$
\begin{aligned}
\mathrm{TF}_{f} & =\max \left(2 \sin (\delta)-\frac{1}{a}(p+a-2 f) \sin (\delta), 0\right) \\
& =\max \left(\left(2 f+1-\frac{p}{a}\right) \sin (\delta), 0\right) .
\end{aligned}
$$

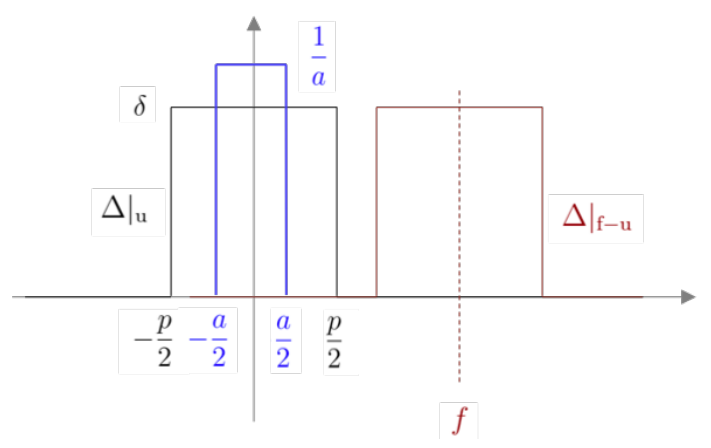

Fig. B.1. ZWFS mask in one dimension with a uniform modulation representing the PSF (in blue), the centered mask $\Delta \mid u$ (black), and the shifted mask $\left.\Delta\right|_{f-u}$ (red).

Case 2. The dot is smaller than the PSF: $p<a$.

Case 2.1: $f \geq p$.

We again have $\left.\Delta\right|_{u}=\delta$ and $\left.\Delta\right|_{f-u}=0$. Consequently, the transfer function equals:

$$
\begin{aligned}
\left.\mathrm{TF}\right|_{f} & =\frac{2}{a} \int_{-a / 2}^{a / 2} \mathrm{~d} u \sin \left(\left.\Delta\right|_{u}-\left.\Delta\right|_{f-u}\right) \\
& =\frac{2}{a} \int_{-p / 2}^{p / 2} \mathrm{~d} u \sin (\delta)=\frac{2 p}{a} \sin (\delta) .
\end{aligned}
$$

Case 2.2: $f<p$.

The $\left.\Delta\right|_{u}-\left.\Delta\right|_{f-u}$ still depends on $f$. Equation (A.2) becomes:

$$
\begin{aligned}
\mathrm{TF}_{f} & =\frac{2}{a} \int_{-a / 2}^{a / 2} \mathrm{~d} u \sin \left(\left.\Delta\right|_{u}-\left.\Delta\right|_{f-u}\right) \\
& =\frac{2}{a} \int_{-f}^{f} \mathrm{~d} u \sin (\delta)=\frac{2 f}{a} \sin (\delta) .
\end{aligned}
$$

The plot corresponding to these results is given earlier in the Letter (Fig. 1).

\section{Appendix C: Pyramid WFS class}

We derived Eq. (A.2) for the PyWFS class once more. The phase of the filtering mask now equals:

$\left.\Delta\right|_{u}=\alpha|u|$.

Here, $\alpha$ equals $2 \pi \theta / \lambda$, where $\theta$ is the pyramid apex angle and $\lambda$ is the sensing wavelength. Equation (A.2) then becomes:

$\left.\mathrm{TF}\right|_{f}=\left.2 \int_{\mathbb{R}} \mathrm{d} u \omega\right|_{u} \sin (\alpha(|u|-|f-u|))$.

In the sine, we have:

$\alpha(|u|-|f-u|)=\left\{\begin{array}{ll}-\alpha f & u \in]-\infty ; 0] \\ \alpha(2 u-f) & u \in] 0 ; f[ \\ \alpha f & u \in[f ; \infty[\end{array}\right.$.

Moreover, $\omega$ is centro-symmetric so it is possible to reduce the integration interval:

$\left.\mathrm{TF}\right|_{f}=\left.2 \int_{-f}^{f} \mathrm{~d} u \omega\right|_{u} \sin (\alpha(|u|-|f-u|))$. 


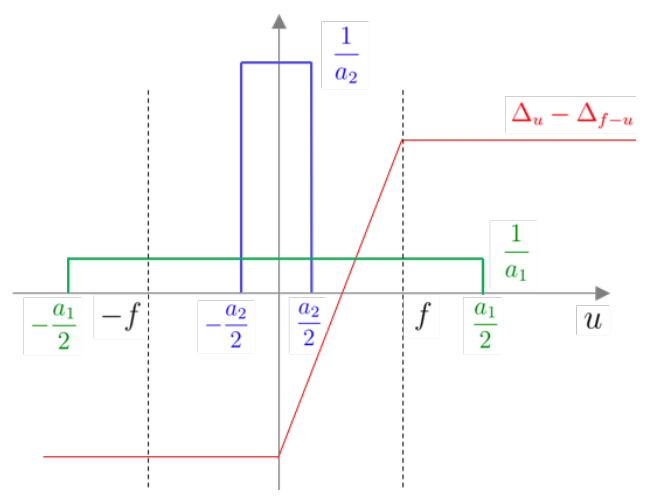

Fig. C.1. In blue (resp. green): case of a weighting function with a small (resp. large) characteristic length; in red, the function of Eq. (C.3).

Figure C.1 allows us to visualize the functions involved in the previous equation. Notably, it appears that the value of the characteristic length of the weighting function over two (i.e., $a / 2$, the modulation radius when this device is active) plays a major role in the integration and can be seen as a cutoff frequency. Equation (C.4) becomes:

$$
\begin{aligned}
\left.\mathrm{TF}\right|_{f}= & \frac{2}{a}\left[\int_{-\min (a / 2, f)}^{0} \sin (-\alpha f) \mathrm{d} u+\int_{0}^{\min (a / 2, f)} \sin (\alpha(2 u-f)) \mathrm{d} u\right] \\
= & \frac{2}{a}\left[\frac{\sin (\alpha \min (a / 2, f)) \sin [(\min (a / 2, f)-f) \alpha]}{\alpha}\right. \\
& -\min (a / 2, f) \sin (\alpha f)] .
\end{aligned}
$$

Finally, we get the transfer function of the pyramid class depending on its two optical parameters, namely the pyramid apex angle and the weighting function characteristic size.

$$
\left.\mathrm{TF}\right|_{f}=\left\{\begin{array}{ll}
\frac{-2 f \sin (\alpha f)}{a} & f \leq a / 2 \\
\operatorname{sinc}(\alpha a / 2) \sin [(a / 2-f) \alpha]-\sin (\alpha f) & f>a / 2
\end{array} .\right.
$$

We can now use this formula to explain the sensitivity with respect to the spatial frequencies of the PyWFS class.

Firstly, we are interested in the influence of the apex angle parameter on the sensitivity. In other words, we studied the difference between classical and flattened pyramids. To do so, we assumed that the modulation is inactive. Consequently, the parameter $a$ is related to the PSF size. Considering a pupil diameter of $\mathrm{D}$, a sensing wavelength of $\lambda$, and a imaging focal of $f_{\mathrm{oc}}$, we have:

$a=\frac{\lambda f_{\mathrm{oc}}}{D}$.

To distinguish between flattened and classical pyramids, we just have to identify the limit apex angle $\theta_{\text {limit }}$ that allows the pupil images to be totally separated. It can be linked with the pupil diameter and the imaging system focal length via the following formula:

$\theta_{\text {limit }}=\frac{D}{f_{\text {oc }}}$.

Consequently, if $\theta$ is below $\theta_{\text {limit }}$, there is an overlap of the pupil images and the pyramid is therefore a flattened one, whereas a

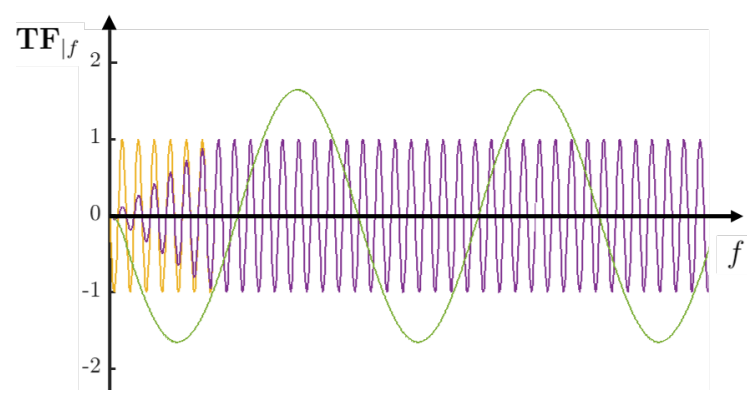

Fig. C.2. Graphical visualization of the transfer function, i.e., Eq. (C.7), for the PyWFS configurations of Fig. 6: non-modulated PyWFS in yellow, modulated PyWFS in purple, and FPyWFS in green.

$\theta$ above $\theta_{\text {limit }}$ implies a complete separation of the pupil images and thus a classical pyramid. If we use the $a$ and $\alpha$ variables, these two cases can be summarized in the following way:

$a \alpha<\pi \quad$ Flattened pyramid

$a \alpha \geq \pi \quad$ Classical pyramid.

Such a distinction allows us to explain why the sensitivity of the flattened pyramid may be larger in absolute value than that of the classical pyramid. As a matter of fact, the sinc function in Eq. (C.7) may be significant for some frequencies when $a \alpha$ is small (i.e., for flattened pyramids). Keeping in mind that the sensitivity is linked with the TF via the convolution product of Eq. (8), this shows why the FPyWFS sensitivity may reach 2 when the classical pyramid optimally attains 1 . Moreover, the small $\alpha$ implies large oscillations with respect to the spatial frequencies (see the green curve in Fig. C.2). This is relevant regarding the observed sensitivity, which does oscillate (dotted green curve in Fig. 6).

By contrast, if $a \alpha$ is large (i.e., if pupil images are completely separated), the sinc function can be neglected and the transfer function summarized as:

$\left.\mathrm{TF}\right|_{f}=\left\{\begin{array}{ll}\frac{-2 f \sin (\alpha f)}{a} & f \leq a / 2 \\ -\sin (\alpha f) & f>a / 2\end{array}\right.$.

This function can be seen as the transfer function of the classical pyramid. We notice that it oscillates more rapidly than the flattened pyramid one (yellow and purple curves in Fig. C.2). However, these oscillations disappear when we look at the corresponding sensitivity curves (Fig. 6). Such a peculiarity can be explained by Eq. (8): to get the sensitivity curve, the transfer function is convoluted with the PSF, which in this case is larger than the oscillation period. As a result, the transfer function is smoothed and the sensitivity follows the TF envelope.

Concerning this envelope, we can observe two regimes. The first one goes from the null spatial frequency to the modulation radius $a / 2$; it is linear with $f$. The second corresponds to spatial frequencies above the modulation radius; it is constant and equal to 1 . We identify here the typical behavior of the classical pyramid (modulated or not) with its two regimes, slope and phase sensors, separated by a cutoff frequency corresponding to the modulation radius (Vérinaud 2004).

The convolutional approach therefore demonstrates its capability to describe the sensitivity of pyramid sensors. It indeed allows a unique formula to be obtained, which explains both the enhanced and oscillating sensitivity of the FPyWFS and the dual behavior slope-phase sensors of the classical modulated PyWFS. 